\title{
Die Sicherstellung der gesundheitlichen Versorgung in einem Flächenland - Erfahrungen aus Brandenburg
}

\section{BETTINA BAUMGARDT}

Bettina Baumgardt ist Leiterin des Referats 21, Grundsatzfragen der Gesundheitspolitik, Gesundheitsziele, Gesundheitsberichterstattung, neue medizinische Versorgungsstrukturen im Ministerium für Umwelt, Gesundheit und Verbraucherschutz des Landes Brandenburg

In Brandenburg wird es vor allem in den berlinfernen Regionen zunehmend schwerer, frei werdende Arztstellen wieder zu besetzen. Die Nachfrage nach qualifizierten Fachkräften im Gesundheitswesen wächst. Die künftige Sicherstellung der flächendeckenden medizinischen Versorgung einer älter werdenden und zahlenmäßig abnehmenden Bevölkerung kann nur mithilfe von gemeinsamen Aktivitäten aller Beteiligten im gegliederten System der medizinischen Versorgung und unter Einbeziehung der infrastrukturellen Rahmenbedingungen gelingen.

\section{Schwer zu versorgende Regionen - wie geht man mit diesem Begriff um?}

Das politische Leitbild von der Gleichwertigkeit der Lebensverhältnisse wird in Deutschland vor dem Hintergrund der Folgen des demografischen Wandels gerade wieder neu diskutiert. In den 60er Jahren der alten Bundesrepublik wurde ein Stadt-Land-Gefälle, auch im Sinne eines Modernisierungsdefizits, konstatiert, dem es auch mit Mitteln der Infrastrukturpolitik zu begegnen galt. In den 70er Jahren richtete sich der Blick dann auf Disparitäten sowohl in regionaler als auch in sozialer Hinsicht. In den 80er Jahren wurde vom NordSüd-Gefälle gesprochen, als Folge von in den Regionen unterschiedlichen Voraussetzungen für und Anpassungsstrategien an den ökonomischen Wandel. Mit der deutschen Wiedervereinigung wurden dann intensive Aktivitäten auf allen genannte Ebenen erforderlich. Seit den 2000 er Jahren wird wieder verstärkt eine Diskussion um das Verständnis von Gleichwertigkeit in Anerkennung der Unterschiede geführt. Unterschiede auf einer horizontalen Ebene beschreiben Benachteiligungen, denen mit geeigneten politischen Maßnahmen begegnet werden muss. Die vertikale Ebene verweist auf Verschiedenheiten, die nicht zuerst oder nicht zwangsläufig Vor- oder Nachteilsbehaftet sind.

Wer aber muss und kann handeln, wenn sich Unterschiede - hier Unterschiede in der gesundheitlichen Versorgung - unangemessen nachteilig für

1 Dies ist natürlich eine extrem verkürzte Darstellung der Debatte. Ich danke meinen Kollegen von der Gemeinsamen Landesplanung für verschiedene Unterweisungen in diesem Feld sowie Herrn Dr. Göschel vom deutschen Institut für Urbanistik für angeregten Austausch. Eventuelle Unrichtigkeiten in der Wiedergabe liegen an mir. 
Menschen in bestimmten Regionen auswirken und wann ist Unangemessenheit zu konstatieren? Mit dieser Frage sehen sich Länder und auch Kommunen in zunehmendem Maße konfrontiert. Und für die gesundheitliche Versorgung ist vor allem die erste Frage, die Frage nach der konkreten Verantwortung, nicht so ohne weiteres zu beantworten. Das gegliederte und selbstverwaltete Gesundheitswesen in Deutschland mit seinen verschiedenen Sicherstellungsaufträgen und dem hochkomplexen Gefüge aus Selbstverwaltungs- und legislativer Steuerung gibt hierauf oft schwer handhabbare Antworten.

Fragen nach der „Letztverantwortung in der Daseinsvorsorge“, diesem juristisch und verwaltungswissenschaftlich so kontrovers diskutierten Begriff, stellen sich in den „schwer zu versorgenden Regionen“ neu. Die Diskussion führt bis in die Frage, auf was sich der

\section{Fragen nach der „Letztverantwortung in der Daseinsvorsorge" stellen sich in den „schwer zu versorgenden Regionen" neu.}

Leistungsanspruch des Einzelnen gegenüber dem Gemeinwesen beziehen kann, auf die Leistung also solche (Personenbezogen) oder auf die Gewährleistung am (Wohn-)Ort (Raumbezogen).

Auf die zweite Frage, jener nach den Kriterien für Angemessenheit in den Unterschieden, komme ich später noch zurück.

\section{Neue Länder als Labor}

Die neuen Bundesländer können von allen hier beschriebenen Betrachtungsebenen viele Lieder singen. Hier waren in kürzester Zeit enorme wirtschaftliche und soziale Veränderungen zu bewältigen - mit negativen wie positiven Folgen. Eine Folge ist die enorme Veränderung in der Besiedlungsstruktur durch den tiefen Geburteneinbruch in den 90er Jahren und die hohe Abwanderung: In Brandenburg, bzw. in den neuen Bundesländern zeigen sich schon seit geraumer Zeit bestimmte Entwicklungen besonders schnell und besonders ausgeprägt, die sich inzwischen teilweise bundesweit abzeichnen. Dazu gehört die wachsen- de Disparität zwischen städtischen und ländlichen Regionen und die demografische Entwicklung. Manche sprechen daher von den neuen Ländern als von einer Art Labor, in dem sich bestimmte Entwicklungen - aber auch darauf abgestimmte Maßnahmen - relativ früher oder auch ausgeprägter als andernorts beobachten lassen. Folgendes Beispiel veranschaulicht dies: das Bundesministerium für Verkehr, Bauwesen, Städtebau und Raumordnung fördert schon seit mehreren Jahren Modellvorhaben der Raumordnung (MORO), die ausgewählte Regionen in ländlichen Räumen darin unterstützen, sich innovativ den infrastrukturellen Herausforderungen des demografischen Wandels zu stellen. Von den in der laufenden Förderperiode bundesweit ausgewählten 21 Modellregionen liegen 9 in den neuen Bundesländern, 4 allein in Brandenburg. Alle Brandenburger Modellregionen haben das Thema gesundheitliche und soziale Versorgung in diesem Zusammenhang in den Fokus genommen.

Bis zum Jahr 2030 wird die Bevölkerungszahl im Land Brandenburg gegenüber 2010 mit 2,51 Mio. Einwohnern voraussichtlich um ca. 10 Prozent zurückgehen. Dabei wird die Bevölkerung im Berliner Umland leicht zunehmen und in den Berlin fernen Landesteilen stark abnehmen. Hinzu kommen die Wanderungsverluste gegenüber den alten Bundesländern, die auch weiterhin überproportional jüngere Frauen betreffen. Im Berlin fernen Raum wird der Bevölkerungsrückgang anhalten und sich ab 2019 weiter verschärfen. Die Bevölkerungsdichte nimmt von derzeit 60 auf 49 Einwohner je $\mathrm{km}^{2}$ im Jahr $2030 \mathrm{ab}$. Zu den bisher 31 Ämtern und amtsfreien Gemeinden mit einer Bevölkerungsdichte unter 25 $\mathrm{EW} / \mathrm{km}^{2}$ kommen bis 2030 weitere 22 hinzu. Bis 2025 werden etwa $40 \%$ der Gesamtfläche Brandenburgs vergleichbar dünn besiedelt sein. Die Bevölkerung in den Oberzentren der Städte Brandenburg a. d. Havel, Cottbus, und Frankfurt/Oder wird bis 2030 zwischen 8 und $12 \%$ abnehmen. Unter den vier Brandenburger Oberzentren wird allein für die Stadt Potsdam ein Bevölkerungsanstieg von rund $19 \%$ prognostiziert. Bereits heute leben in weiten Teilen des Landes nur bis zu 30 Einwohner pro qkm, davon rechnerisch etwa 2 Kinder und 3 Jugendliche. Wurde 2010 jeder fünfte Brandenburger zu den Senioren gerechnet, wird 2030 bereits mehr als jeder dritte über 65 Jahre alt sein, bei, so hoffen wir, weiter steigender Lebenserwartung. Die absolute Zahl der Hochaltrigen wird sich bis 2030 gegenüber 2010 verdoppeln. ${ }^{2}$

\section{Infrastrukturpolitik und gesundheitliche Versorgung zusammen denken - sowohl planerisch als auch konkret}

Auf diese enormen Besiedlungsveränderungen muss raumplanerisch reagiert werden und Gesundheitspolitik kann sinnvoller weise nur gemeinsam mit dieser Perspektive gedacht werden. Das sehen die Akteure in Brandenburg und reagieren darauf. Ein Beispiel: Die Bedarfsplanung in der vertragsärztlichen Versorgung erfolgte in Brandenburg wie überall in den zurückliegenden 20 Jahren gemäß der Bedarfsplanungsrichtlinie Ärzte auf der Grundlage der Landkreise bzw. Kreisfreien Städte. Mit der Vergrößerung der Landkreise nach der Kommunalreform 2003 in Brandenburg vergrößerte sich für die KV Brandenburg als Inhaberin des Sicherstellungsauftrages für die vertragsärztliche Versorgung auch das Problem einer sachgerechten Steuerung der ärztlichen Niederlassung. Die betrachteten Räume waren schlicht zu groß geworden. Den erheblichen Disparitäten innerhalb dieser Räume konnte planerisch nicht mehr begegnet werden. Die Kassenärztliche Vereinigung Brandenburg hat sich deshalb früh und nachhaltig für eine Weiterentwicklung der Betrachtungsgrundlagen eingesetzt. Die für Infrastruktur und für das Gesundheitswesen zuständigen Ressorts der Landesregierung arbeiten in diesem Feld seit 2009 intensiv zusammen. Der gemeinsame Landesausschuss der Ärzte und Krankenkassen in Brandenburg hat auf seiner Sitzung am 24. November 2010 die ambulante Versorgungssituation in Brandenburg erstmals auf der Grundlage der von der Gemeinsamen Landesplanungsabteilung Berlin-Bran-

2 AfS Berlin Brandenburg \& LBV (2012). Bevölkerungsprognose für das Land Brandenburg. 2011 bis 2030. A I 8 - 11; LBV (2012) Bevölkerungsvorausschätzung 2011 bis 2030. Ämter und amtsfreie Gemeinden des Landes Brandenburg 
denburg definierten 46 Mittelbereiche $^{3}$ untersucht. Die guten Erfahrungen der Zusammenarbeit zwischen Landesplanung und den Akteuren des Gesundheitswesens in Brandenburg sind auch in die aktuelle Weiterentwicklung der Bedarfsplanungsrichtlinie auf Bundesebene eingeflossen.

Die Kommunen haben einen erheblichen Einfluss darauf, wie die Bürger - einschließlich der potentiellen, künftigen medizinischen Fachkräfte - die Lebensqualität am Wohnort einschätzen. Denn vielfach ausschlaggebend für die Wahl des Lebens- und Arbeitsortes von Fachkräften sind Faktoren wie zum Beispiel die vorherige Kenntnis/Heimatverbundenheit, die Infrastruktur (insbesondere Schulen, Kindergärten, Arbeitsmöglichkeiten für die Partnerin/ den Partner), Arbeitsbedingungen und z. T. auch Bezahlung. Kommunen unterstützen Ärzte und ihre Familien bei der Ansiedlung und Bereitstellung von Praxisräumen, auch in Zweigstellen und Unterkünften für Weiterbildungsassistenten und Praktikanten. Hier gibt es einerseits einen Wettbewerb zwischen Kommunen um Fachkräfte. Andererseits ist ein arbeitsteiliges und abgestimmtes Vorgehen zwischen Nachbargemeinden bzw. Mittelzentren und Umland nötig, damit eine regionale Ansiedlungsförderung „aus einer Hand“ erfolgreich sein kann. Als Leistungen der Kommunen ist hier auch ein abgestimmtes Angebot von regelmäßigen und/oder speziellen Shuttle-Diensten zur Kompensation eines ausgedünnten traditionellen $\mathrm{Nah}$ verkehrs zu nennen. Der Deutsche Städte- und Gemeindebund hat hier „Landarzttaxis" (zum Transport zu und von der Praxis) genannt. Außerdem werden Praxisfilialen in kleinen Gemeinden, die an bestimmten Tagen besetzt sind und mobile Dienste wie beispielsweise eine rollende Zahnarztpraxis als Anknüpfungspunkte auch kommunaler Initiativen genannt. Sowohl der Deutsche Landkreistag als auch der Deutsche Städte- und Gemeindebund haben die Notwendigkeit betont, dass - wie in Brandenburg - Politik, KV, Krankenkassen, Ärztekammer, Landkreise und Gemeinden gemeinsam Ideen entwickeln müssen, um einer drohenden Verschlechterung der medizinischen Versorgung im ländlichen Raum wirksam begegnen zu können. ${ }^{4}$

Die Ansiedlung von Ärztinnen und
Ärzten in Regionen mit deutlichen Versorgungsproblemen kann in Brandenburg sowohl im Rahmen der Förderung zur Verbesserung der Grundversorgung auf dem Lande als auch in kleineren Städten auch mit EU-Mitteln unterstützt werden. Aus dem Europäischen Landwirtschaftsfonds für die Entwicklung ländlicher Räume (ELER), aus der Gemeinschaftsinitiative URBAN II (Förderperiode 2000-2006) und aus dem Europäische Fonds für Regionale Entwicklung (EFRE) - Förderrichtlinie „Nachhaltige Stadtentwicklung " Teil KMU wurden Einrichtungen der medizinischen Versorgung in Brandenburg gefördert. Das Programm LEADER, in dem lokale Aktionsgruppen mit den Akteuren vor Ort maßgeschneiderte Entwicklungskonzepte für ihre Region erarbeiten, bietet zudem den Rahmen, um soziale Infrastrukturen in regionale Konzepte einzubinden. Über die Brandenburger ILE/LEADER Richtlinie 2012 wurde bspw. einer Zahnärztin aus Templin (Vorhaben aus der LAG Uckermark/RAK Naturparkregion Uckermärkische Seen) - eine mobile Zahnarztpraxis gefördert. Zu Fördermöglichkeiten aus dem Europäischen Landwirtschaftsfonds für die Entwicklung ländlicher Räume (ELER) und der Richtlinie für die integrierte ländliche Entwicklung (ILE) haben wir 2009 eine gemeinsame Informationsveranstaltung des für Infrastruktur und ländliche Entwicklung zuständigen Ministeriums und des Gesundheitsministeriums mit den Sozialdezernenten und LEADER-Regionalmanagern der mit Versorgungsproblemen betroffenen Landkreise/ Regionen und der Kassenärztlichen Vereinigung Brandenburg durchgeführt. Über die Fördermöglichkeiten des Europäischen Fonds für Regionale Entwicklung (EFRE) - Förderrichtlinie „Nachhaltige Stadtentwicklung“ hinzuweisen ist grundsätzlich Aufgabe der am Programm beteiligten Städte. Darüber hinaus wurde die Kassenärztliche Vereinigung im Rahmen der Vorbereitung der Bewilligungsverfahren einbezogen. Zudem berichtet das Gesundheitsministerium im Rahmen von Spitzengesprächen zur künftigen medizinischen Versorgung im ländlichen Raum allen Beteiligten im Lande über Fördermöglichkeiten aus den Strukturfonds.

In Brandenburg hat man sich darauf

verständigt, ein gemeinsames Landesgremium für sektorenübergreifende Versorgungsfragen im Sinne des $\mathbb{S} 90 \mathrm{a}$ SGB V einzurichten. Ein entsprechendes Landesgesetz befindet sich zur Zeit in der Ressortabstimmung. Die Länder hatten vom Bund mehr und weitreichendere Kompetenzen gefordert. Diese und einige weitere Gestaltungsmöglichkeiten der Länder in der Gesundheitspolitik sind Ergebnis eines Kompromisses,

Es kommt darauf an, Fragen von Gesundheitsversorgung und Fragen von Infrastrukturentwicklung gemeinsam zu betrachten.

der mit dem Versorgungsstrukturgesetz umgesetzt wurde. Gleichwohl soll dieses neue Instrument in Brandenburg genutzt werden. Denn die Steuerung von Versorgung und die Entwicklung zukunftsfähiger Konzepte, insbesondere für strukturkritische Regionen, erfordert eine sektorenübergreifende systematische Betrachtung auf Landesebene. Dabei kommt es auch darauf an, Fragen von Gesundheitsversorgung und Fragen von Infrastrukturentwicklung gemeinsam zu betrachten. Regionale Versorgungsbedürfnisse, raumordnerische Aspekte und Perspektiven der demografischen Entwicklung müssen berücksichtigt werden. Für unterschiedliche Räume und Gegebenheiten werden angepasste Konzepte benötigt, damit alle Menschen angemessen erreicht werden können.

Wir leben länger und werden gesünder alt, aber sowohl die Anforderungen an die gesundheitliche Versorgung als

3 Zur räumlichen Ordnung der höherwertigen Daseinsvorsorge wird landesplanerisch ein flächendeckendes System Zentraler Orte festgelegt, die als räumlich-funktionale Schwerpunkte komplexe Funktionen für ihr jeweiliges Umland erfüllen. Die Grundversorgung wird innerhalb der amtsfreien Gemeinden und Ämter abgesichert. Die Verflechtungsbereiche der Mittelzentren und der Oberzentren bilden in Brandenburg insgesamt 46 Mittelbereiche, die bei der Festlegung über mindestens 30.000 Einwohner verfügten, so dass für mittelzentrale Einrichtungen eine ausreichende Tragfähigkeit besteht. (vgl. www.mil.brandenburg.de)

4 Deutscher Landkreistag September und Oktober 2010, Medizinische Versorgung im ländlichen Raum; Deutscher Städte- und Gemeindebund April 2010, Ärztliche Versorgung im ländlichen Raum 
auch die Bedingungen in den Regionen in Deutschland ändern sich erheblich

Die Lebenserwartung ist ein Indikator für den Gesundheitszustand in einer Gesellschaft. Das heißt, hier spiegeln sich in einer einzigen Kennziffer verschiedene Einflussfaktoren auf die Gesundheit, wozu genetische Dispositionen, individuelle und kollektive Verhaltensweisen, regionale Ernährungsgewohnheiten, soziale Lage und die Leistungsfähigkeit des medizinischen Versorgungssystems gehören. Für die Entwicklung in den vergangenen Jahren lässt sich für Brandenburg eine erfreuliche Bilanz ziehen. Die Lebenserwartung der Brandenburger Bürger und Bürgerinnen ist stetig gestiegen. Ost- und Westdeutschland unterschieden sich direkt nach der Wiedervereinigung in der Mortalität (Sterblichkeit) und damit verbunden der durchschnittlichen Lebenserwartung, in der Morbidität (häufige chronische Krankheiten), in zahlreichen Gesundheitsrisiken und in der Qualität der gesundheitlichen Versorgung. Gesundheit existiert nicht unabhängig von sozialen und ökonomischen Rahmenbedingungen. Diese haben sich seit 1989 auch in Brandenburg grundlegend verändert. Nach 20 Jahren gemeinsamer Entwicklung haben sich die Unterschiede in den meisten Bereichen deutlich verringert oder sogar angeglichen. Nur selten erfolgte die Angleichung auf einem schlechteren Niveau als dem Ausgangsniveau in den neuen Bundesländern, etwa beim Auftreten von Allergien und dem Anteil der Raucherinnen. Neben diesem positiven Fazit zeigen die Analysen allerdings nach wie vor starke regionale Unterschiede in der Verteilung von Gesundheitschancen in Deutschland. Unterschiede in der Gesundheit und im Gesundheitsverhalten ergeben sich oftmals aus einer ungleichen Verteilung von Bildung, Arbeitslosigkeit, Einkommen und Privatvermögen in den Regionen, Städten oder Gemeinden ${ }^{5}$. Durch die Gesundheitsberichterstattung des Landes werden die Zusammenhänge zwischen Gesundheit und sozialer Lage und Geschlecht systematisch und kontinuierlich für Brandenburg dargestellt.

\section{Wissen wir genug um sachgerecht zu steuern?}

Und hier komme ich auf die eingangs gestellte Frage zurück: welche Unter- schiede (in der gesundheitlichen Versorgung) sind hinzunehmen, welche nicht? Und wie kommen wir zu angemessenen Kriterien dafür? Um die richtige Weichenstellung für eine gerechte und sachgerechte Steuerung der Ressourcen im Gesundheitswesen wird im Rahmen regelmäßiger Reformdebatten gerungen. Leistungserbringer erfüllen Aufgaben in einem äußerst komplexen gegliederten System, davon war schon die Rede. Sie liefern Qualität, sie haben Interessen, sie reagieren auf Vergütungsanreize. Es gibt Konkurrenz um Ressourcen zwischen Sektoren, Berufsgruppen und Regionen. Es gibt selbstverständlich auch Konkurrenz um die Steuerungskompetenzen selbst, siehe Versorgungsstrukturgesetz und die Gestaltungsmöglichkeiten der Länder. Das Steuerungsinstrumentarium wird stetig weiterentwickelt, es wird nachjustiert und verfeinert und dabei auch stetig komplexer. Argumente für

\section{Die Aufbereitung und Bereitstellung von Leistungsdaten für die Versorgungsforschung in Deutschland kommt erst langsam auf den Weg.}

eine Steuerung in die eine oder andere Richtung sind leichter $\mathrm{zu}$ finden und $\mathrm{zu}$ widerlegen, wenn man über geeignete Daten verfügt. Hier liegt - vor allem auch aus Landessicht - ein Problem: Für die Beplanung der unterschiedlichen Sektoren werden teilweise unterschiedliche Daten zugrunde gelegt. Ganz überwiegend handelt es sich um Daten aus dem Leistungsgeschehen selbst. Gesundheitsökonomisch wird oft die These von der angebotsinduzierten Nachfrage in der medizinischen Versorgung vertreten: Dort, wo viele Leistungserbringer sind, werden auch viele Leistungen in Anspruch genommen. Das Zentralinstitut der Kassenärztlichen Bundesvereinigung hat im Rahmen des Fachsymposiums „Regionale Besonderheiten in der Versorgungs- und Versichertenstruktur am 25.05. 2011 in Berlin Daten vorgestellt, die diese These stützen. Die geeignete Aufbereitung und Bereitstellung von Leistungsdaten für die Versorgungsforschung in Deutschland kommt jedoch erst langsam auf den Weg. Vor allem mit Blick auf die Aussagefähigkeit zu regionalen Unterschieden wird von wissenschaftlicher Seite eine qualifiziertere Befassung gefordert. ${ }^{6}$

Warum ist das aus der Brandenburger Perspektive so bedeutsam? Wenn die These stimmt, dass dort, wo viele Leistungserbringer sind, auch viele Leistungen in Anspruch genommen und abgerechnet werden (Patientenwanderungen nicht eingerechnet) so bildet sich das einerseits in den Daten ab, die prospektiven Bedarfsplanungen (stationär, vertragsärztlich) zugrunde liegen. Andererseits dienen dieselben Daten der Ermittlung künftiger Ressourcenbedarfe, die durch den Morbiditätsorientierten Risikostrukturausgleich auch regional abgebildet und verteilt werden. In Brandenburg war die Zahl der Leistungserbringer im Bundesvergleich schon immer unterdurchschnittlich und sie wird wohl absehbar weiter sinken, während die Zahl der Menschen mit einem hohen Morbiditätsrisiko durch den demografischen Wandel zunächst steigt. Prof. Wasem hat anlässlich der besagten Tagung meine Frage, ob aus diesem Mechanismus nicht auch ein Kellertreppeneffekt (weniger Leistungserbringer - weniger Leistungsgeschehen - sinkende Mittel aus dem Morbi-RSA) für strukturkritische Regionen abzuleiten sei, mit ja beantwortet.

Es besteht also aus Ländersicht, vor allem aus der Sicht solcher Länder, die wie Brandenburg mit den beschriebenen Strukturentwicklungen zu kämpfen haben, ein hohes Interesse an einer verbreiterten Datengrundlage für die Bedarfsplanung, an Versorgungsforschung und an Aussagen zu regionalen Unterschieden. Deshalb hat Brandenburg im Gesetzgebungsverfahren zum Versorgungsstrukturgesetz nach einem Ansatzpunkt für diese Perspektive gesucht, zunächst in der ambulanten Bedarfsplanung. Wir wollten erreichen, dass die Krankheitslast nicht nur mit Leistungsdaten im Sinne des $\mathbb{} 303$ und der $\mathbb{S} \mathbb{3} 303$ a bis $303 \mathrm{f}$ (Datentransparenz) bestimmt werden soll sondern mittelfristig auch epidemiologische Daten, die unabhängig von den

\footnotetext{
5 Vgl. Robert Koch Institut (2009), Beiträge zur Gesundheitsberichterstattung des Bundes, 20 Jahre nach dem Fall der Mau-er: Wie hat sich die Gesundheit in Deutschland entwickelt?

6 Vgl. Petition 37108 „Forschung - Regionalmerkmal zur Unterstützung der Versorgungsforschung"vom 17.10.2012
} 
in Anspruch genommenen Leistungen gewonnen wurden, zur Ermittlung der Krankheitslast herangezogen werden. Damit sollte die Entwicklung eines planungsrelevanten Datenpools für die künftige Bedarfsplanung ermöglicht werden, welcher sich sowohl an Daten aus der Leistungsinanspruchnahme orientiert, als auch Aussagen zur Krankheitslast, unabhängig von den in Anspruch genommenen Leistungen zulässt. Denn erst die Gegenüberstellung beider Sachverhalte ermöglicht Aussagen zu Über-, Fehl- und Unterversorgung. Die Bundesregierung mochte dem Bundesrat an dieser Stelle nicht folgen.

\section{Hilft ein „Landärztegesetz“ weiter?}

Die Frage, „wie viel ,Land' darf drin sein in der Gesundheitspolitik? " hat bei der Debatte um das Versorgungsstrukturgesetz eine zentrale Rolle gespielt. Die Initiative für einige wesentliche Aspekte dieses Gesetzes ist maßgeblich von den Bundesländern ausgegangen. Dafür gibt es gute Gründe, die Bettina am Orde und Hartmut Reiners ausführlich beschrieben haben. ${ }^{7}$

In der öffentlichen Wahrnehmung hat jedoch eine andere Frage dominiert. Das Versorgungsstrukturgesetz wird ja teilweise auch als „Landärztegesetz“ bezeichnet. Das klingt gut - aber was ist dran? Die künftige Sicherstellung der medizinischen Versorgung in ländlichen Regionen wird nur gelingen, wenn die vorhandenen Ressourcen klug und gemeinsam eingesetzt werden können und

\section{Die vom SVR dringend empfohlene Stärkung der nicht- ärztlichen Gesundheitsberufe muss immer wieder auf die Tagesordnung.}

wenn ein wirksames Umverteilungsmoment dafür sorgen hilft, dass bundesweit einerseits Überversorgung abgebaut wird und andererseits die medizinischen Leistungen in strukturschwächeren ländlichen Regionen angemessen finanziert sind. Von den Mechanismen der Ressourcensteuerung war weiter oben schon die Rede. Ein wirklich wirksames Umverteilungsmoment fehlt bislang. Zudem liegt der Fokus zu stark im fach- ärztlichen Bereich, der nun in einer Art drittem Sektor geregelt wird (spezialfachärztliche Versorgung nach $\mathbb{} 116 \mathrm{~b}$ SGB V). Eine Tätigkeit dort wird, auch durch dieses Gesetz, immer attraktiver. Die hausärztliche Versorgung wird nicht ausreichend gestärkt. Und die vom Gesundheits-Sachverständigenrat dringend empfohlene Stärkung der nicht-ärztlichen Gesundheitsberufe muss immer wieder auf die Tagesordnung.

\section{Trotzdem immer wieder richtig - Initiativen auf Landesebene}

Aber auch wenn die bundespolitischen Rahmenbedingungen nicht in jeder Hinsicht zufriedenstellend sind, liegen die Potenziale immer zuerst in der $\mathrm{Zu}$ sammenarbeit. Und für die gibt es in Brandenburg eine gute Tradition. Regelmäßige Gespräche auf Spitzenebene, angeregt durch die Ministerin für Gesundheit, zeigen Einigkeit zwischen den wichtigen Akteuren im Brandenburger Gesundheitswesen. Im zurückliegenden Jahr hat das Brandenburgische Gesundheitsministerium dem Fachausschuss des Landtages ein ausführliches Papier über Grundlagen, Handlungsfelder und Maßnahmen zur künftigen Sicherstellung der medizinischen Versorgung vorgelegt ${ }^{8}$. Ein durchaus nicht zu unterschätzender Nutzten dieses Papiers für Interessierte auf der Landesebene lag auch in der verständlichen Beschreibung der verschiedenen Zuständigkeiten und Bezüge, einerseits auf Bundes- und Landesebene und andererseits zwischen den verschiedenen Akteure der Selbstverwaltung. Ein nicht geringer Teil von Ressourcen in der politischen Diskussion auf Landesebene fließt oft in die Aufklärung von Missverständnissen über Zuständigkeiten und Kompetenzen. Für einen Dialog, der alle einbezieht - von den Partnern im Gesundheitswesen über die kommunale und die landesplanerische Ebene bis hin zu Patientinnen und $\mathrm{Pa}$ tienten, Bürgerinnen und Bürgern und der sie vertretenden Parteien, die landesund kommunalpolitisch agieren und engagiert mittun - braucht es zunächst eine Verständigungsgrundlage. Auch deshalb wurde das Papier sowohl mit allen Beteiligten aus dem Gesundheitswesen als auch im Fachausschuss des
Landtages ausführlich erörtert. Auch die ressortübergreifende Zusammenarbeit wird damit erleichtert, neue Perspektiven der Kooperation werden eröffnet.

Wichtige Handlungsfelder für die künftige Sicherstellung der gesundheitlichen Versorgung in Brandenburg sind im Papier ebenfalls beschrieben. Auf einige soll hier beispielhaft eingegangen werden

\section{Fachkräftesicherung und ärztliche Nachwuchsgewinnung}

In Brandenburg bemühen sich alle Beteiligten seit längerem um die Nachwuchssicherung von Fachkräften z.B. durch die Kampagne www.arzt-in-brandenburg.de, die das Land bereits 2006 gemeinsam mit allen Beteiligten ins Leben gerufen hat. Auch das Thema Kooperation und flexible Arbeitszeiten spielt in Brandenburg traditionell eine wichtige Rolle. Brandenburg besitzt keine eigene medizinische Fakultät. Über die Verwaltungsvereinbarung zur zweiten Programmphase des Hochschulpaktes 2020 erhält das Land Berlin aufgrund seiner - auch mit Blick auf Brandenburger Bedarfe - überproportional vorgehaltenen Studienplätze in der Medizinerausbildung 10 Mio. € zusätzlich zulasten der Programmpauschale für die neuen Länder. Im Rahmen von Gesprächen zwischen dem brandenburgischen Gesundheitsministerium und der Charité - Universitätsmedizin Berlin werden Möglichkeiten einer verstärkten Kooperation zwischen den brandenburgischen Krankenhäusern und der Charité erörtert, um Medizinstudierenden der Charité bereits während ihrer Ausbildung hinreichende Gelegenheiten zu eröffnen, das Land Brandenburg und die hiesigen Beschäftigungsmöglichkeiten und -rahmenbedingungen kennenzulernen. Unabhängig vom spezifischen System, innerhalb dessen die medizinische Versorgung und die Nachwuchsgewinnung erfolgen, bedarf es ergänzend auch eines interdisziplinären Zugangs. Im Ergebnis der Empfehlungen der Fachkräftestudie Berlin-Brandenburg 2010 ist die künftige Sicherstellung der medizinischen

\footnotetext{
7 Am Orde, Bettina / Reiners, Hartmut (2011) Das Versorgungsstrukturgesetz und die Erwartungen der Länder. Gesundheits- und Sozialpolitik 56

8 www.mugv.brandenburg.de/Gesundheit/ Gesundheitsziele, Versorgung
} 
Versorgung im ländlichen Raum das Leitprojekt des Gesundheitsministeriums zur strategischen Ausrichtung des Maßnahmenplans Fachkräftesicherung in Brandenburg. Genannt sei hier auch das Brandenburger Qualifizierungsprojekt für zugewanderte Ärztinnen und Ärzte in Zusammenarbeit mit der OttoBennecke-Stiftung. Das Projekt konnte sich als Modell für ein künftiges bundesweites Qualifizierungsangebot für Einwanderer mit Hochschulabschluss aufstellen. Die Lehrgangskosten wurden vom Land übernommen, zudem wurden EU-Mittel eingesetzt (Landesprogramm „Qualifizierung und Arbeit für Brandenburg“)

\section{Integrierte und fachübergreifende Versorgungsmodelle}

Für medizinische Versorgungszentren hat sich Brandenburg seit Beginn der 1990er Jahre stark gemacht. Im Einigungsvertrag war lediglich ein Bestandsschutz für die im Beitrittsgebiet bestehenden staatlichen Gesundheitseinrichtungen (Polikliniken, Ambulatorien) festgelegt. Die Landesregierung Brandenburg hat sich für ihren Fortbestand mit großem Nachdruck eingesetzt und ihren Erhalt unter dem Begriff Gesundheitszentren auch mit Modellförderung unterstützt. Diese Versorgungsform wurde dann unter dem Namen Medizinische Versorgungszentren 2004 bundesweit zugelassen.

Seit September 2009 arbeiten die KV Brandenburg und die AOK Nordost gemeinsam mit der BARMER GEK in einer - bislang bundesweit einmaligen - Partnerschaft zur Sicherung der medizinischen Versorgung insbesondere in den ländlichen Regionen ihres Bundeslandes in der Initiative „IGIB - Innovative Gesundheitsversorgung in Brandenburg“"zusammen. Sie haben sich unter anderem dem Abbau der Grenzen zwischen ambulant und stationär, neuen integrierten Versorgungskonzepten, einer effizienteren Arznei- und Heilmittelversorgung sowie Konzepten für die Versorgung chronisch Kranker verschrieben. In diesem Rahmen hat die KV Brandenburg das Modell eines fachübergreifenden ärztlichen Kooperationszentrums - KVRegioMed zur Optimierung der medizinischen Versorgung in ländlichen Regionen entwickelt. KVRegioMed soll einerseits die ambulante Versorgung mit der stationären Grundversorgung verzahnen und es andererseits ermöglichen, dass Ärzte vor allem der grundversorgenden Fachrichtungen flexibel Sprechstunden in Filialnetzen anbieten können. Fallmanager, Heil- und Hilfsmittelerbringer sowie Pflegedienste sollen hinreichend einbezogen werden. Zudem könnten regional organisierte Hol- und Bringdienste die Strukturen verbessern.

Der Beitrag der Krankenhäuser zur künftigen Sicherstellung der medizinischen Versorgung in Brandenburg zielt ebenfalls auf eine gute regionale Zusammenarbeit. Mit der Entwicklung neuer Berufsbilder, der Planung und Erprobung neuer Versorgungsmodelle und der Prozessoptimierungen innerhalb der Kliniken werden im stationären Bereich innovative Wege beschritten hin zu einer optimierten Versorgung. An der ambulanten Notfallversorgung beteiligen sich die Krankenhäuser sektorenübergreifend bspw. durch regionale Notfallversorgungsverbünde oder durch Notfallsprechstunden des kassenärztlichen Notdienstes im Krankenhaus.

Ein weiteres Beispiel ist das seit Januar 2008 existierende prosper-Netz in der Lausitz mit über 260 Haus- und Fachärzten sowie dem Carl-Thiem-Klinikum in Cottbus und dem Krankenhaus Spremberg. Über 24.000 Versicherte der Knappschaft und der DAK haben sich bei prosper Lausitz eingeschrieben.

Es gibt in Brandenburg zudem viele Beispiele für ganz konkrete innovative Formen der Zusammenarbeit auf regionaler Ebene, z.B. zwischen Öffentlichem Gesundheitsdienst und Krankenhaus im Feld der Kindergesundheit oder

\section{Es gibt in Brandenburg viele Beispiele für ganz konkrete innovative Formen der Zusammenarbeit auf regionaler Ebene.}

zwischen Kassenärztlicher Vereinigung, Kommunen und Krankenkassen für einen regionalen Patientenbus. Auch Mittel aus EU-Fonds (s.o.) werden für neue Formen medizinischer Versorgung im ländlichen Raum genutzt, wie zum Beispiel eine mobile Zahnarztpraxis.

\section{Arztentlastende Fachkräfte}

Wir setzen uns dafür ein, Ärztinnen und Ärzte durch den Ausbau von Telemedizin oder Modelle wie AGneS zu entlasten. AGnES steht für arztentlastende, gemeindenahe, E-Healthgestützte, s systemische Intervention Die Akzeptanz des AGnES-Konzeptes (entwickelt durch das Institut für Community Medicine der Universität Greifswald) war bei den am brandenburger Modellprojekt teilnehmenden Hausärzten und Patienten sehr hoch. Die Projektergebnisse veranlassten den Gesetzgeber schließlich, diese Versorgungsform in die Regelversorgung zu überführen. Aus der „AGnESFachkraft" wurde zum 1. April 2009 die nicht-ärztliche Praxisassistentin.

\section{Telemedizin}

Das Gesundheitsministerium ist gemeinsam mit Krankenkassen, der Kassenärztlichen Vereinigung, der Landeskrankenhausgesellschaft, der Landesärztekammer, dem Verband der Medizinischen Versorgungszentren, dem Verband der privaten Kliniken und Pflegeeinrichtungen und der Industrie- und Handelskammer Potsdam im Gespräch, wie telemedizinische Anwendungen zur künftigen Sicherstellung der medizinischen Versorgung im ländlichen Raum besser eingesetzt und Hindernisse beseitigt werden können. Hierbei erfolgt eine enge Zusammenarbeit mit der Telemedinitiative Brandenburg. Projekte werden gemeinsam mit der TSB Medici umgesetzt, die vom Ministerium für Wirtschaft und Europaangelegenheiten gefördert wird und im „Masterplan Gesundheitsregion Berlin-Brandenburg “ die Zuständigkeit für die Weiterentwicklung der Telemedizin auch für das Land Brandenburg hat. Die Verbesserung der hausärztlichen Versorgung durch telemedizinische Verfahren und die stärkere Vernetzung der fachärztlichen Kompetenz zwischen den Krankenhäusern mittels Telemedizin ist ein interessantes Standortargument für Brandenburg. Beispielhaft sei hier das FONTANE-Projekt genannt, das im strukturschwachen ländlichen Raum in Nordbrandenburg mittels Telemedizin an der Verbesserung der Betreuungsqualität für Patienten mit Herzkreislauferkrankungen arbeitet. Das Projekt konn- 
te sich im Wettbewerb des Bundesministeriums für Bildung und Forschung "Gesundheitsregionen der Zukunft“ gegen mehr als 80 weitere Projekte als Sieger durchsetzen. In Anlehnung und in Kooperation mit dem Fontaneprojekt will das Ministerium für Umwelt, Gesundheit und Verbraucherschutz mit Partnern aus dem Land und der Charité im Jahr 2011 ein landesweites telemedizinisches Netzwerk für Patienten mit schwerer Herzinsuffizienz realisieren. Ebenfalls mit Unterstützung des Ministeriums für Umwelt, Gesundheit und Verbraucherschutz will sich ein Netzwerk zur besseren Versorgung von akuten Schlaganfallpatienten gründen. Mittels Telekooperation zwischen Krankenhäusern soll es ermöglicht werden, diese Patienten noch schneller und besser $\mathrm{zu}$ versorgen.

\section{Ausblick}

Die Akteure in Brandenburg teilten im Wesentlichen die Diagnosen und aufgezeigten Handlungsmöglichkeiten im genannten Papier über Grundlagen, Handlungsfelder und Maßnahmen. Im September dieses Jahres wurde das $\mathrm{Pa}$ pier in seinem Zahlenwerk aktualisiert. Eine vollständige Überarbeitung und Anpassung ist für Ende 2013 / Anfang 2014 geplant, denn dann werden neue rechtliche Rahmenbedingungen auf Landes- auf Bundes- und auf europäischer Ebene wirksam werden, die auf die Sicherstellung der medizinischen Versorgung in Brandenburg wesentlichen Einfluss haben. In Brandenburg wird in 2013 die landesrechtliche Grundlage für ein Gemeinsames Landesgremium im Sinne des $\mathbb{9}$ 90a SGB V geschaffen. In Brandenburg wird zudem aktuell die Krankenhausfinanzierung umgestellt, die dazu erforderliche Änderung des Krankenhausentwicklungsgesetzes Brandenburg (KHEG) wird 2013 in Kraft treten. Durch den Gemeinsamen Bundesausschuss werden im neuen Jahr die Rahmenbedingungen für die ambulante vertragsärztliche Bedarfsplanung einerseits und für die spezialfachärztliche Versorgung nach $\$ 116$ b SGB V andererseits neu festgelegt. Im Bereich der Anerkennung von ausländischen Abschlüssen hat sich bundesrechtlich durch das Berufsqualifikationsfestsetzungsgesetz (BQFG) einiges getan. Hierzu wird es in 2013 die erforderlichen Umsetzungsverordnungen vom Bund geben.

Die Berufsanerkennungsrichtlinie der

edarfsgerechte Anpassung Systems gesundheitlicher orgung wird zukünftig als zuvor von der

\section{Kooperation der wichtigsten ure abhängen.}

EU befindet sich aktuell in der Revision und wird voraussichtlich 2013 wesentliche Veränderungen erfahren.

\section{Die gute Nachricht zum Schluss: Die Menschen werden gesünder alt als jemals zuvor, das gilt auch für Brandenburg.}

In den nächsten Jahren wächst die relative und absolute Zahl von Menschen in höherem und höchstem Alter. Die gesundheitliche Versorgung mit ihren Hauptakteuren, Berufen, Strukturen und Abläufen wird sich verändern. Die bedarfsgerechte Anpassung des Systems gesundheitlicher Versorgung wird mehr als zuvor von der Kooperation der wichtigen Akteure abhängen. Die Umsetzung wird dann erfolgreich sein, wenn alle Beteiligten gemeinsame Ziele verfolgen und der Austausch über gute Beispiele weiter wächst.

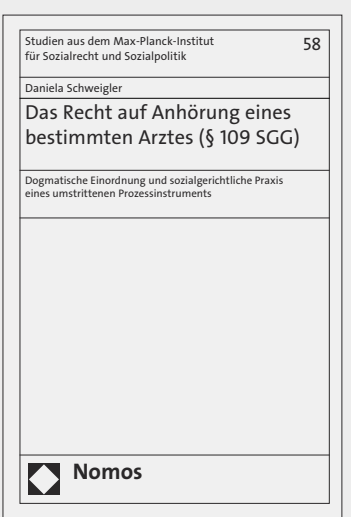

Das Recht auf Anhörung eines bestimmten Arztes ( $\$ 109$ SGG) Dogmatische Einordnung und sozialgerichtliche Praxis eines umstrittenen Prozessinstruments Von Daniela Schweigler 2013, 322 S., brosch., 78,-€ ISBN 978-3-8487-0177-3

(Studien aus dem Max-PlanckInstitut für Sozialrecht und Sozialpolitik, Bd. 58)

Die Arbeit widmet sich dem Antragsrecht auf Anhörung eines bestimmten Arztes im sozialgerichtlichen Verfahren nach § 109 SGG. Der dogmatischen Einordnung des Antragsrechts in die Grundsätze und Prinzipien des Verfahrensrechts folgt die empirische Auswertung einer bundesweiten Untersuchung sozialgerichtlicher Verfahren.

www.nomos-shop.de/20352

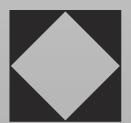

